\title{
Efecto de la orientación emprendedora en las exportaciones de las empresas colombianas ${ }^{1}$
}

\section{Impact of the entrepreneur guidence in the colombian exports companies}

dOI: http://dx.doi.org/10.17981/econcuc.39.1.2018.02

Artículo de investigación. Fecha de recepción: 05/12/2017 Fecha de aceptación: 27/02/2018

Edgar Julián Gálvez-Albarracín

Universidad del Valle. Cali (Colombia).

edgar.galvez@correounivalle.edu.co

Miguel Fernando Reyes-Velasco

Universidad del Valle. Cali (Colombia). mifreyes@gmail.com

Augusto Rodríguez-Orejuela

Universidad del Valle. Cali (Colombia).

augusto.rodriguez@correounivalle.edu.co

Para citar este artículo:

E. Gálvez, M. Reyes-Velasco y A. Rodríguez. "Efecto de la orientación emprendedora en las exportaciones de las empresas colombianas" Económicas CUC, vol. 39, no. 1, pp. 27-40, 2018 DOI: http://dx.doi.org/10.17981/econcuc.39.1.2018.02

\section{Resumen}

En el actual entorno de competencia globalizada es importante que las organizaciones consideren incursionar en mercados externos, por ello, este trabajo busca determinar cómo influye la orientación emprendedora de las empresas colombianas en su disposición hacia los mercados internacionales. El estudio es cuantitativo y correlacional; para ello se empleó el modelo de regresión lineal. Los resultados denotan que la primera variable influye positiva y muy significativamente sobre la segunda, especialmente en el caso de las empresas exporadoras no tradicionales. Se concluye que cuanto más orientados hacia el emprendimiento estén los directivos de las empresas, éstas tendrán mayor propensión a exportar.

Palabras clave: orientación emprendedora; mercado internacional; empresas exportadoras tradicionales; empresas exportadoras no tradicionales

\section{Summary}

Nowadays in the competitive globalized environment is relevant to organizations to consider entering abroad markets, therefore, this research project pursues to determine the influence of the entrepreneur guidance in the Colombian exports companies related to tendencies to foreign exchanges, the methodology used by researchers of this work was quantitative and correlative as well a pattern of linear regression was employed. With respect to the obtained results these have shown that the first variable has an explicit and relevant effect on nontraditional exports companies. To summarize it can be said that the entrepreneurship orientation applied by the management staffs consequently will provide outstanding paths to exportations.

Keywords: entrepreneur guidance, abroad markets, traditional export companies, nontraditional export companies

${ }^{1}$ Este artículo es producto del proyecto "La orientación al mercado internacional y los resultados empresariales de las Born Global en Colombia” realizado por el Grupo de Investigación en Marketing de la Universidad del Valle. Cali (Colombia). 


\section{Introducción}

La internacionalización es un tema de gran interés en el ámbito empresarial y económico (Santos y García, 2016; Giménez y Piedecausa, 2015; Múnera, Arroyave, Vanegas, Morales y Botero, 2015; Vanegas, Restrepo y González, 2015; Ruesga y Bichara, 2007), definiéndose frecuentemente como la adaptación tanto de los procesos de la organización como sus productos y servicios al contexto global, independiente del ámbito geográfico donde se produzca (Maldonado, García, Martínez, Aguilera, González y Vivanco, 2013; Giménez, 2010; Calof y Beamish, 1995). Según Ruzzier, Hisrich y Antoncic (2006), es fundamental acumular ciertos recursos para lograrlo, y, especialmente, desarrollar capacidades como la de orientación al mercado internacional (OMI).

Madsen, Rasmussen y Servais (2001) señalan, por su parte, al mercado internacional como un ambiente idóneo para la puesta en marcha de emprendimientos; lo mismo manifiestan Lu, Liang, Shan y Liang (2015), especialmente cuando se tiene una estructura organizacional adecuada. Sin embargo, la mayoría de empresas comienzan a exportar sólo después de haber desarrollado sus mercados internos, por lo que se les conoce como "exportadoras tradicionales", mientras que otras, desde su inicio, se comprometen en actividades de comercio exterior. A estas últimas se les llama exportadoras no tradicionales o "Born Global" (Knight y Cavusgil, 1996). En este contexto, la OMI permite a las empresas identificar mejor las oportunidades y amenazas comerciales que ofrece el mundo (Lohrke, Franklin y Kothari, 2015; Álvarez y López, 2004; Diamantopolus y Cadogan, 1996).

Por su parte, en la búsqueda de nuevos negocios y de mercados externos puede ser muy útil el emprendimiento corporativo como estrategia que implica la realización de actividades emprendedoras y arriesgadas por parte de las organizaciones (Bedoya, Toro y Arango, 2017; Baruah y Ward, 2015;
Desai, 2015; Varela e Irizar, 2009; Trujillo y Guzmán, 2008; Garzón, 2004; Antoncic y Hsrich, 2003). Consecuentemente con esto, la orientación emprendedora o al emprendimiento (OE), a través de la asunción de riesgos calculados por parte de los directivos y de procesos de innovación, facilita a las empresas la creación o mejoramiento de productos y estrategias que la adaptan mejor al entorno local o externo, logrando así nuevas redes y oportunidades de desarrollo (Flores, Ojeda, Lee y Ramírez, 2016; Martins, Rialp, Rialp y Aliaga-Isla, 2015; Ferreira, Azevedo y Fernández, 2011; Li, Liu, Duan y Li, 2008; Zahra, Jennings y Kuratko, 1999).

Algunos estudios como los de Baker, Grinstein y Harmancioglu (2015), Ferreira et al. (2011), Blesa y Ripolles (2005), Matzuno y Mentzer (2002) y Covin y Slevin (1991) han mostrado la positiva relación entre la OE de las empresas y su desarrollo, incluido el de su OMI; no obstante lo anterior, los trabajos se enfocan mayormente en mercados de países industrializados, por lo cual se deben adelantar más investigaciones en el tema y que incluyan a las naciones emergentes (Steenkamp y Burgess, 2006; Wiklund y Shephard, 2005).

Según lo señalado, la pregunta que intenta resolver este trabajo es: ¿cómo influye la orientación emprendedora (OE) de los directivos de las empresas en la orientación de la firma hacia los mercados internacionales (OMI)? Para tratar de resolverla, se tomó una muestra de 308 empresas exportadoras, incluyendo tradicionales y "Born Global" (o no tradicionales), todas ubicadas en Colombia.

El documento se estructura así: primero se presenta la fundamentación teórica correspondiente, las investigaciones previas encontradas y se plantean las hipótesis a contrastar. Luego, se indica la metodología usada, se discuten los resultados, y por último, se plantean las conclusiones, limitaciones y posibles futuras líneas de investigación. 


\section{Fundamentación teórica}

Orientación emprendedora $(\mathrm{OE})$. El concepto de la OE surge de los campos de la estrategia y el emprendimiento (Arroyo, Bojica y Fuentes, 2007); es así que Lumpkin y Dess (1996) la definen como el estilo gerencial que utiliza para la toma de decisiones, métodos y prácticas que reflejan la asunción de riesgos, la innovación, la proactividad y la agresividad competitiva. Según Kyriakopoulos, Meulember y Nilsson (2004), la cultura emprendedora en la empresa tiene, además, características como mecanismos de relacionamiento y flexibilidad, e incluye aspectos como el apropiado sistema de recompensas, recursos para nuevos proyectos, estructura organizacional de soporte y tolerancia a las equivocaciones (Gálvez y García, 2011; Wood, 2004).

Las empresas emprendedoras desarrollan proyectos que se anticipan a las oportunidades del entorno y a las iniciativas de los competidores, asumiendo importantes dosis de riesgo calculado y altas expectativas de recompensa. Por el contrario, las empresas que no lo son tienden a tomar una posición reactiva y adversa al riesgo, siguiendo e imitando a los competidores (Antoncic, 2003; Barringer y Bluedorn, 1999).

Orientación al mercado internacional (OMI). Son pocos los sectores empresariales que no se han visto sometidos a un intenso proceso de apertura y globalización (Blonqvist, Hurmelinna, Nummela y Saarenketo, 2008), de tal manera que, en 1996, Diamantopoulus y Cadogan plantearon un nuevo enfoque para la orientación al mercado desde lo externo, denominandolo orientación al mercado internacional y centrándolo en las exportaciones. Para Cadogan, Diamantopoulus y De Mortanges (1999), la apertura y gestión de exportaciones incluye actividades típicas del mercadeo como lo es la identificación de: los clientes y competidores internacionales, los cambios en los mercados extranjeros y los programas de apoyo gubernamental en el país de origen, al igual que de otros factores adicionales, como las diferencias culturales entre los países que participan en el intercambio.

Es así que teniendo como referente las investigacones de Kohli y Jaworski (1990), se plantean las siguientes fases en la orientación al mercado internacional:

Generación de la inteligencia internacional. Son las actividades usadas para detectar mercados de exportación y entender a sus clientes, competidores, reglamentaciones comerciales y los cambios en todos estos. Actuar exitosamente en los entornos complejos de los mercados foráneos dependerá de la habilidad de la empresa para generar información de las variables que puedan influir en su desempeño.

Diseminación de la inteligencia internacional. Es la difusión en la organización de la información producida en la fase anterior. Cuanto más grande sea la empresa será necesario usar mecanismos más refinados para la diseminación de los datos generados.

Capacidad de respuesta internacional. En línea con las fases previas, cuanto mejor sea la generación y difusión de información, mayor será la eficacia en el diseño y aplicación de respuestas a los retos del mercado externo (Cadogan, Diamantopoulos y Siguaw, 2002). Según Dimantopoulos y Cadogan (1996), hay factores de cada componente de la OMI a ser especialmente considerados: en la generación de inteligencia es relevante la experiencia en los mercados externos, tener información depurada y no depender de terceras partes para ello; en la diseminación de los datos, se deben eliminar barreras como la sobrecarga o el exceso de filtros, y la respuesta al mercado debe ser racional, según la política de comunicación organizacional; finalmente, según estos autores, los mecanismos de coordinación deben evitar problemas entre las subsidiarias y la casa matriz. 
Empresas "Born Global”. El término "Born Global” fue acuñado por la consultora McKinsey (McKinsey y Co., 1993) en un estudio para el Consejo de Fabricación Australiana. Según Madsen et al. (2001), una "Born Global" es una empresa que inicia su proceso de exportaciones en los primeros tres años después de su fundación y que tiene un porcentaje de exportaciones del $25 \%$ o más respecto al total de sus ventas. Oviatt y McDougall (1994) definen la nueva empresa internacional como aquella que, desde su inicio, busca obtener significativas ventajas competitivas mediante la venta de sus productos en múltiples países, y donde la gerencia está comprometida con la internacionalización (Harveston, Ben y Davis, 2000).

Es importante destacar que para entender mejor a las "Born Global" se pueden integrar las corrientes de investigación sobre la internacionalización y el emprendimiento (Madsen et al., 2001). Es así que Sharma y Blomstermo (2003) señalan que se caracterizan por ser empresas jóvenes y emprendedoras que aparecen en sectores de alta tecnología y también en convencionales.

Por su parte, Rasmussen y Madsen (2002) definen los siguientes criterios para catalogar a una empresa como tal: 1) no ser representante, filial ni subsidiaria de otra empresa, 2) no ser de naturaleza pública, 3) el $25 \%$, o más, del total de sus ventas debe ser resultado de actividades exportadoras, 4) máximo a los 7 años de fundada debe haber alcanzado los mercados internacionales. Por descarte simple, las empresas exportadoras que no son clasificadas como "Born Global” se consideran "exportadoras tradicionales".

Estudios previos sobre la relación entre orientación emprendedora y orientación al mercado internacional. Es difícil encontrar estudios que aborden esta relación de manera directa, por lo tanto, se presentan las pocas que lo hacen y las que la tratan tangencialmente:
Ferreira et al. (2011) encuentran, mediante regresiones lineales en una muestra de 168 empresas manufactures de Portugal, que la OE influye positivamente en el crecimiento de estas organizaciones $\mathrm{y}$, en particular, sobre su nivel de ventas y de generación de empleo. Los resultados de la investigación publicada por Blesa y Ripolles en el 2005 muestran, mediante ecuaciones estructurales y con una muestra de $222 \mathrm{em}$ presas españolas, que la OE influye positiva y significativamente sobre la orientación al mercado.

En un estudio realizado con 1300 empresas de los Estados Unidos de América, Matzuno y Mentzer (2002) encuentran una relación significativa y positiva entre la $\mathrm{OE}$ y la OMI, siendo especialmente importante dentro de los factores que la generan, la baja aversión al riesgo. Madsen et al. (2001) encuentran en sus investigaciones que las pequeñas y medianas empresas "Born Global" juegan un importante papel en el crecimiento económico de un país, pero que presentan ciertas características que limitan su capacidad exportadora, como el acceso a la información, la tecnología, la poca innovación y las dificultades de acceso a la financiación.

Lumpkin y Dess (1996) encuentran que la OE favorece las oportunidades de negocio e impacta de manera positiva la orientación al mercado, la expansión de la empresa y su progreso tecnológico. Lo anterior centrado en estilos gerenciales innovadores y de escaso temor frente a las variables ambientales.

Hipótesis. A partir del marco teórico presentado y los trabajos empíricos previos señalados, se plantean las siguientes hipótesis a contrastar para el caso colombiano: H1. El nivel de OE y de OMI son significativamente diferentes en las empresas exportadoras tradicionales frente a las "Born Global".

H2. Cuanto más fuerte es la OE de los directivos, mayor es la OMI en las empresas, tanto en las exportadoras tradicionales como en las "Born Global". 


\section{Metodología de investigación}

La muestra estuvo conformada por 308 empresas exportadoras escogidas aleatoriamente de las bases de datos de las siguientes entidades y gremios colombianos: PROEXPORT $^{2}, \mathrm{FENALCO}^{3}, \mathrm{ANALDEX}^{4}$ y CONFECAMARAS ${ }^{5}$, las cuales, en el momento de la investigación, tenían 9213 organizaciones afiliadas; de esta manera se obtuvo un margen de confianza del 95\% y un $5 \%$ de error. Las tablas 1,2 y 3 presentan la conformación de la muestra según la actividad económica de las empresas, su ubicación geográfica y tamaño, respectivamente:

TABLA 1

Distribución de las empresas según su actividad económica

\begin{tabular}{cc}
\hline Sector de la economía & Porcentaje \\
\hline Manufacturero & $70 \%$ \\
Servicios & $3 \%$ \\
Comercio & $5 \%$ \\
Agrícola & $8 \%$ \\
Tecnología & $1 \%$ \\
Otros sectores & $13 \%$ \\
\hline
\end{tabular}

Fuente: Adaptado de Reyes, Rodríguez y Gálvez (2015).

Los datos se recogieron telefónicamente entre enero y mayo del 2013 mediante un cuestionario elaborado con base en la teoría existente y que fue aplicado a los directivos de las organizaciones objeto de la investigación.

\footnotetext{
${ }^{2}$ PROEXPORT: Agencia estatal colombiana para el fomento del comercio exterior. Actualmente ha cambiado su nombre por PROCOLOMBIA.

${ }^{3}$ FENALCO: Federación Nacional de Comerciantes de Colombia

${ }^{4}$ ANALDEX: Agremiación colombiana de entidades dedicadas al comercio exterior

${ }^{5}$ CONFECAMARAS: Federación de Cámaras de Comercio de Colombia
}

TABLA 2

Distribución de las empresas según su ubicación geográfica

\begin{tabular}{cc}
\hline Ciudad & Porcentaje \\
\hline Bogotá & $38 \%$ \\
Medellín & $19 \%$ \\
Cali & $19 \%$ \\
Barranquilla & $5 \%$ \\
Bucaramanga & $2 \%$ \\
Otras ciudades & $17 \%$ \\
\hline
\end{tabular}

Fuente: Adaptado de Reyes et al. (2015).

TABLA 3

Distribución de las empresas según su tamaño ${ }^{6}$

\begin{tabular}{cc}
\hline Tamaño de empresa & Porcentaje \\
\hline Micro empresa & $14 \%$ \\
Pequeña empresa & $30 \%$ \\
Mediana empresa & $32 \%$ \\
Gran empresa & $24 \%$ \\
\hline
\end{tabular}

Fuente: Adaptado de Reyes et al. (2015).

Por su parte, la tabla 4 indica la distribución de la muestra según las empresas se clasifiquen como "Born Global" o exportadoras tradicionales, de acuerdo con la tipología planteada por Madsen et al. (2001). Es importante informar aquí que, a criterio de los investigadores, se utilizó una cantidad parecida de empresas para cada tipo, pero que ello no refleja necesariamente la distribución en la población donde tienden a haber más exportadoras tradicionales.

\footnotetext{
${ }^{6}$ En Colombia, las leyes 590 del 2000 y 905 del 2004 determinan así el tamaño de las empresas: grande: más de 200 trabajadores y más de 30.000 salarios mínimos mensuales legales vigentes (SMMLV); mediana: entre 51 y 200 trabajadores y hasta 30.000 SMMLV; pequeña: entre 11 y 50 trabajadores y hasta 5000 SMMLV; y microempresas: de 1 a 10 trabajadores y un capital de hasta 500 SMMLV.
} 
TABLA 4

Distribución de las empresas según su tipología como exportadoras

\begin{tabular}{cc}
\hline "Born Global" & Exportadoras tradicionales \\
\hline 159 & 149 \\
\hline
\end{tabular}

Fuente: Adaptado de Reyes et al. (2015)

\section{Medición de las variables}

Orientación emprendedora (OE). Para medir la OE se ha utilizado la escala desarrollada por Robinson, Stimpson, Huefner y Hunt (1991), la cual está compuesta por cinco ítems que recogen la percepción de los altos directivos sobre su relación con el ambiente, y especialmente, su capacidad de encontrar oportunidades en él y de afrontar los riesgos consecuentes. Según se muestra en la tabla 5, las preguntas fueron de tipo Likert de siete puntos, correspondiendo el 1 a "totalmente en desacuerdo" y el 7 a "totalmente de acuerdo".

TABLA 5

Escala para la medición de la orientación emprendedora - OE

\section{ORIENTACIÓN EMPRENDEDORA}

Cuál es su grado de acuerdo con las siguientes afirmaciones, siendo 1 totalmente en desacuerdo y 7 totalmente de acuerdo:

Para tener éxito en los negocios debe dedicar cada día algún tiempo al desarrollo de nuevas oportunidades.

Gran parte de su tiempo se enfoca en buscar varias ideas de nuevos de negocios.

Usted se siente orgulloso cuando mira los resultados que ha obtenido en sus actividades de negocio.

Usted tiene la experiencia suficiente como para estar seguro en la forma en que usted procede en los negocios.

Usted cree que su habilidad para relacionarse con la gente le ha permitido crear muchas oportunidades de negocio.

Fuente: Adaptado de Robinson et al. (1991).
Orientación al mercado internacional $(O M I)$. Esta variable se revisó a través del constructo propuesto por Cadogan, Diamantoupulus y De Mortanges (1999), que se conforma por tres fases o dimensiones: generación de información de mercados internacionales; diseminación de información de mercados internacionales; y respuesta al mercado internacional. Las tablas 6,7 y 8 muestran las escalas usadas para cada dimensión:

TABLA 6

Escala para la medición de la generación de información de mercados internacionales

Usando una escala de 1 a 7 , donde 1 es Totalmente en desacuerdo y 7 Totalmente de acuerdo, califique cada una de las siguientes afirmaciones en relación con lo que ocurre en su empresa:

Revisan periódicamente el posible efecto de cambios en el entorno exportador (por ejemplo, la tecnología, la regulación).

Recopilan información sobre los clientes internacionales para ofrecer un mejor servicio.

Detectan rápidamente los cambios fundamentales en el entorno exportador (por ejemplo, la tecnología, la economía regulatoria).

Recopilan información sobre los clientes internacionales para detectar los cambios en sus preferencias.

Fuente: Adaptado de Reyes et al. (2015).

TABLA 7

Escala para la medición de la diseminación de la información de mercados internacionales

Usando una escala de 1 a 7 , donde 1 es totalmente en desacuerdo y 7 totalmente de acuerdo, califique cada una de las siguientes afirmaciones en relación con lo que ocurre en su empresa.

El personal de mercadeo se reúne con otras áreas para detectar las necesidades futuras de los clientes internacionales.

Los datos sobre la satisfacción de sus clientes internacionales son distribuidos por toda la firma de forma regular.

Cuando un departamento descubre algo importante sobre la competencia en mercados extranjeros, suele ser muy rápido en alertar a otros departamentos.

Cuando pasa algo importante en sus mercados de exportación, se informa rápidamente a otros departamentos.

Fuente: Adaptado de Reyes et al. (2015). 
TABLA 8

Escala para la medición de la respuesta a los mercados internacionales

Usando una escala de 1 a 7 , donde 1 es totalmente en desacuerdo y 7 totalmente de acuerdo, califique cada una de las siguientes afirmaciones en relación con lo que ocurre en su empresa.

Son rápidos en decidir cómo responder a los cambios de precios de sus competidores en el mercado de exportación.

Reaccionan en corto tiempo a los cambios en las necesidades de productos o servicios de sus clientes extranjeros.

Revisan periódicamente sus esfuerzos de desarrollo de productos para asegurarse de que están en línea con lo que los clientes extranjeros quieren.

$\mathrm{Si}$ su mejor competidor pone en marcha una intensa campaña dirigida a sus clientes extranjeros, se pone en práctica una respuesta de inmediato.

Son rápidos para adaptar los cambios que se presentan en el contexto de los negocios internacionales.

Fuente: Adaptado de Reyes et al. (2015).

Empresas exportadoras tradicionales o "Born Global". Para ser clasificadas como "Born Global" en este trabajo, las empresas debieron cumplir los requisitos propuestos por Rasmussen y Madsen (2002). Las que no lo hicieron se clasificaron como exportadoras tradicionales.

Modelo teórico. El siguiente es el modelo teórico en el cual se basa el análisis inferencial que se propone en este trabajo:

$$
\mathrm{OMI}_{\mathrm{i}}=\mathrm{b}_{0}+\mathrm{b}_{1 \mathrm{i}} \mathrm{OE}_{\mathrm{i}}+\varepsilon_{\mathrm{i}}
$$

Donde $\mathrm{OMI}_{\mathrm{i}}$, como variable dependiente, representa el grado de orientación al mercado internacional para las empresas "Born Global" o exportadoras tradicionales, consolidando y promediando a los tres factores que la conforman (generación de información de mercados internacionales, diseminación de la información y respuesta a los mercados), y que se presume está en función de la variable independiente OE (orientación emprendedora), manifestado por los directivos de las respectivas empresas.

Validez de constructos. Mediante la contrastación del estadístico Alpha de Cronbach, la tabla 9 confirma la fiabilidad de los constructos usados. Esto brinda seguridad para el estudio de los fenómenos y permite continuar con el procesamiento de las variables propuestas.

\section{Resultados y discusión}

La tabla 10 presenta los resultados descriptivos del trabajo. Puede observarse de manera general que las medias de todas las varialbes son superiores al valor intermedio de la escala Likert usada $(4,0)$, lo que demuestra que las "Born Global" y las empresas exportadoras tradicionales colombianas tienen unos aceptables niveles de OE y de OMI, y que en ambos tipos de empresas su OMI es más baja que su OE.

TABLA 9

Verificación de fiabilidad de los constructos

\begin{tabular}{|c|c|c|c|c|c|}
\hline \multirow[b]{2}{*}{ Variables } & \multicolumn{2}{|c|}{ "Born Global" } & \multicolumn{2}{|c|}{ Exportadoras tradicionales } & \multirow[b]{2}{*}{$\begin{array}{l}\text { Elementos o } \\
\text { preguntas }\end{array}$} \\
\hline & $\begin{array}{l}\text { Alpha de } \\
\text { Cronbach }\end{array}$ & $\begin{array}{l}\text { Número de } \\
\text { casos }\end{array}$ & $\begin{array}{l}\text { Alpha de } \\
\text { Cronbach }\end{array}$ & $\begin{array}{l}\text { Número de } \\
\text { casos }\end{array}$ & \\
\hline Generación & 0,776 & \multirow{4}{*}{159} & 0,835 & \multirow{4}{*}{149} & 4 \\
\hline Diseminación & 0,910 & & 0,834 & & 4 \\
\hline Respuesta & 0,898 & & 0,840 & & 5 \\
\hline $\begin{array}{c}\text { Orientación } \\
\text { emprendedora (OE) }\end{array}$ & 0,790 & & 0,750 & & 6 \\
\hline
\end{tabular}

Fuente: Elaboración propia. 
De igual manera, se encuentra que de los factores que constituyen la OMI, el que presenta menor valor de media es la diseminación, lo que indica que es un factor que requiere especial cuidado, principalmente en las "Born Global” donde fue apenas de 4,84; así mismo, el factor que tiene mayor valor es el de respuesta a los cambios del entorno con 5,27 en las "Born Global" y 5,36 en las exportadoras tradicionales.
Por su parte, la tabla 11 indica que si bien las medias de las variables analizadas son todas mayores en las exportadoras tradicionales, al no ser estas diferencias significativas $(p=0,32$ para OMI y $p=0,943$ para $\mathrm{OE}$ ), no es posible probar la primera hipótesis de esta investigación en cuanto a que en Colombia "el nivel de OE y de OMI son significativamente diferentes en las exportadoras tradicionales frente al de las 'Born Global”'.

TABLA 10

Estadisticos descriptivos

\begin{tabular}{|c|c|c|c|c|c|c|}
\hline $\begin{array}{l}\text { Tipo de } \\
\text { empresa }\end{array}$ & Variable & Media & Mínimos & Máximos & $\begin{array}{l}\text { Desviación } \\
\text { típica }\end{array}$ & $\mathrm{N}$ \\
\hline \multirow{5}{*}{$\begin{array}{l}\text { "Born } \\
\text { Global" }\end{array}$} & Orientación emprendedora (OE) & 5,76 & 2 & 7 & 0,833 & \multirow{5}{*}{159} \\
\hline & Generación & 5,24 & 1 & 7 & 1,27 & \\
\hline & Diseminación & 4,84 & 1 & 7 & 1,69 & \\
\hline & Respuesta & 5,27 & 1 & 7 & 1,32 & \\
\hline & $\begin{array}{l}\text { Orientación al Mercado } \\
\text { Internacional (OMI) }\end{array}$ & 5,13 & 1 & 7 & 1,17 & \\
\hline \multirow{5}{*}{$\begin{array}{l}\text { Exportadoras } \\
\text { tradicionales }\end{array}$} & Orientación emprendedora (OE) & 5,77 & 3 & 7 & 0,87 & \multirow{5}{*}{149} \\
\hline & Generación & 5,28 & 1 & 7 & 1,36 & \\
\hline & Diseminación & 5,11 & 1 & 7 & 1,43 & \\
\hline & Respuesta & 5,36 & 1 & 7 & 1,16 & \\
\hline & $\begin{array}{l}\text { Orientación al Mercado } \\
\text { Internacional (OMI) }\end{array}$ & 5,26 & 1 & 7 & 1,11 & \\
\hline
\end{tabular}

Fuente: Adaptado de Reyes et al. (2015).

TABLA 11

Comparación de medias para exportadoras tradicionales y "Born Global"

\begin{tabular}{|c|c|c|c|c|c|c|c|}
\hline & \multicolumn{7}{|c|}{ Prueba para la igualdad de medias } \\
\hline & \multirow[t]{2}{*}{$\mathrm{T}$} & \multirow{2}{*}{ Gl } & \multirow{2}{*}{$\begin{array}{c}\text { Sig. } \\
\text { (bilateral) }\end{array}$} & \multirow{2}{*}{$\begin{array}{l}\text { Diferencia } \\
\text { de medias }\end{array}$} & \multirow{2}{*}{$\begin{array}{l}\text { Diferencia de } \\
\text { error estándar }\end{array}$} & \multicolumn{2}{|c|}{$\begin{array}{c}\text { Confianza } \\
\text { de la diferencia } \\
\end{array}$} \\
\hline & & & & & & Inferior & Superior \\
\hline $\mathrm{OE}$ &,- 997 & 306 &, 320 &,- 12983 & , 13022 &,- 38607 & ,12642 \\
\hline OMI &,- 072 & 306 &, 943 &,- 00695 & 09691 &,- 19765 &, 18375 \\
\hline
\end{tabular}

Fuente: Elaboración propia. 
Resultados inferenciales. Debido a que se utilizaron regresiones lineales múltiples como técnica estadística correlacional, se verificó previamente el cumplimiento de los siguientes supuestos que aseguran la fiabilidad de los datos usados: homocedasticidad o varianza constante, no existencia de multicolinealidad, normalidad de la distribución e independencia de los términos (Hair, Anderson, Tatham y Black, 1999).

En esta investigación se fijó como grado de multicolinealidad límite 0,19 para su tolerancia y 5,3 para la inflación de la varianza (VIF), por lo que cualquier variable con tolerancia menor de 0,19 o superior de 5,3 indica elevada multicolinealidad (Hair et al., 1999). Es así que en los modelos se descartó dicha situación. Así mismo, se verificó el supuesto de independencia mediante el estadístico Durvin-Watson donde se denota independencia entre los residuos cuando éste muestra valores de entre 1,5 y 2,5 .

La tabla 12 presenta los cálculos realizados para conocer las relaciones entre la OE y la OMI, tanto en las empresas exportadoras tradicionales como en las "Born Global". El valor de las $F$ indica que los modelos usados son validos globalmente $\left(\mathrm{F}=68.693^{* * *}\right.$ y $\mathrm{F}=47.775^{* * *}$, respectivamente); señalando así que la variable independiente efectivamente influye en la variable dependiente objeto de revisión. Por otra parte, los valores de las $\mathrm{R}^{2}(0,300$ y 0,240 , respectivamente) señalan que los cambios en la OE explican un $30 \%$ y un $24 \%$, según el caso, las variaciones de la OMI en la población estudiada.

Efecto de la orientación emprendedora en la orientación al mercado internacional. Por su parte, los betas $\left(0,552^{* * *}\right.$ y $0,495^{* * *}$, respectivamente) indican que tanto en las "Born Global" como en las empresas exportadoras tradicionales los incrementos en la OE tienen efectos significativos y positivos en la OMI. Esto significa que, en la medida en que los directivos de las empresas se
TABLA 12

Relaciones entre la OE y la OMI

\begin{tabular}{|c|c|c|}
\hline \multirow{2}{*}{$\begin{array}{c}\text { Variable } \\
\text { Dependiente } \\
\text { Variables } \\
\text { Independientes }\end{array}$} & \multicolumn{2}{|c|}{$\begin{array}{l}\text { Orientación al Mercado } \\
\text { Internacional (OMI) }\end{array}$} \\
\hline & $\begin{array}{l}\text { Born } \\
\text { Global }\end{array}$ & $\begin{array}{l}\text { Exportadoras } \\
\text { tradicionales }\end{array}$ \\
\hline $\mathrm{R}^{2}$ Ajustado & 0.300 & 0.240 \\
\hline Durvin-Watson & 1.770 & 1.812 \\
\hline $\mathrm{F}$ & $68.693^{* * *}$ & $47.775^{\text {***}}$ \\
\hline VIF más alto & 1,00 & 1,00 \\
\hline $\begin{array}{c}\text { Orientación } \\
\text { emprendedora } \\
\text { (OE) B } \\
\text { t-student }\end{array}$ & $\begin{array}{l}0.552^{* * *} \\
(8.288)\end{array}$ & $\begin{array}{l}0.495^{* * *} \\
(6.912)\end{array}$ \\
\hline
\end{tabular}

${ }^{* *} \mathrm{p} \leq 0.05 ;{ }^{* * *} \mathrm{p} \leq 0.01$

Fuente: Elaboración propia.

implican más en la búsqueda de oportunidades de negocio y se sienten más seguros de llevarlas a cabo, aumenta la propensión de las organizaciones a profundizar sus procesos de exportación. Estos hallazgos coinciden con los de Ferreira et al. (2011), Blesa y Ripolles (2005), y Matzuno y Mentzer (2002), y permiten probar la segunda hipótesis planteada para esta investigación con respecto a que, en Colombia, cuanto más fuerte es la orientación emprendedora de los altos directivos, mayor es la orientación al mercado internacional, tanto en sus empresas exportadoras tradicionales como en sus "Born Global".

\section{Conclusiones, limitaciones y futuras líneas de investigación}

Los resultados de esta investigación permitieron comprobar la positiva y significativa influencia de la Orientación Emprendedora (OE) en la Orientacion al Mercado Internacional (OMI) de las empresas exportadoras colombianas, siendo las siguientes sus principales conclusiones: 
Al poseer las organizaciones estudiadas un aceptable nivel de OMI, se puede deducir que tienen un buen potencial para aprovechar los múltiples convenios de libre comercio que su país ha establecido con naciones como los Estados Unidos de América, la Unión Europea, Costa Rica, etc.. Sin embargo, para que esto se concrete es altamente deseable que fortalezcan sus habilidades exportadoras y su OE, ya que si bien sus capaciades actuales son aceptables, todavía tienen un amplio margen de desarrollo.

Para mejorar su posición competitiva internacional y aprovechar las oportunidades que trae el acelerado proceso de globalización mundial, las empresas exportadoras colombianas, y especialmente las "Born Global", pueden beneficiarse del efecto impulsor que su OE tiene sobre su OMI. Para esto podrían apoyarse en programas e información que entidades como PROCOLOMBIA y ANALDEX tienen.

Los directivos de las empresas exportadoras colombianas deben revisar urgentemente sus prácticas de comunicación, delegación y empoderamiento, ya que la diseminación de la información generada en la fase de inteligencia internacional se muestra como la principal debilidad de su OMI. A su vez, se percibe que la buena capacidad de respuesta que muestran estas empresas puede ser un factor a su favor para enfrentar las turbulencias que en temas como las tasas de cambio y las normas regulatorias se suelen presentar en el país y en otros de los mercados mundiales.

Los hallazgos de este trabajo son de interés para la academia y para las entidades privadas y públicas de fomento al comercio exterior, ya que les ratifica que deben estimular, en los empresarios y gerentes, la búsqueda de nuevas oportunidades de negocio más allá de las fronteras nacionales. A los directivos de las empresas les permite corroborar que para que sus organizaciones incursionen y/o se sostengan competitivamente en el mercado externo deben comprender las dinámicas cambiantes de los mercados foráneos y enfrentar la incertidumbre y los riesgos que la actividad exportadora conlleva.

Como una limitación del trabajo puede mencionarse el hecho de sólo haber consultado en las organizaciones a los directivos, ya que ello puede generar un sesgo en las respuestas obtenidas. Entre los futuros proyectos que podrían derivarse de esta investigación está replicarla en otros subsectores de la economía, como el de software, cosméticos, turismo de salud, etc.; también podría aplicarse en otras naciones emergentes para observar cómo están las empresas exportadoras de Colombia frente a las de países comparables.

\section{Referencias}

Álvarez, R. y López, R. A. (2004). Orientación exportadora y productividad en la industria manufacturera Chilena. Cuadernos de economía, 41(124), 315343.

Antoncic, B. (2003). Risk taking in intrapreneurship: Translating the individual level risk aversion into the organizational risk taking. Journal of Enterprising Culture, 11(1), 1-23.

Antoncic, B. y Hisrich, R. (2003). Clarifying the intrapreneurship concept. Journal of Small Business and Enterprise Development, 10(1), 7-24.

Arroyo, M. R., Bojica, A. M., y Fuentes, M. (2007). "Orientación emprendedora: una aproximación a la interrelación con la gestión de la calidad total y el desempeño". En: Empresa global y mercados locales. Presentado en XXI Congreso Anual AEDEM, Universidad Rey Juan Carlos. Madrid, España: Escuela Superior de Gestión Comercial y Marketing, ESIC. 
Baker, W. E., Grinstein, A. y Harmancioglu, N. (2015). The Role of Entrepreneurial Orientation in Overcoming Known and Unknown Barriers to Entry in Foreign Markets. En: Deeter-Schmelz, D. $\mathrm{R}$ (Eds), Proceedings of the 2010 Academy of Marketing Science (AMS) Annual Conference (pp. 97-97). Athens, USA: Springer International Publishing.

Barringer, B. R. y Bluedorn, A. C. (1999). The relationship between corporate entrepreneurship and strategic management. Strategic Management Journal, 20(5), 421-444.

Baruah, B. y Ward, A. (2015). Metamorphosis of intrapreneurship as an effective organizational strategy. International Entrepreneurship and Management Journal, 11(4), 811-822.

Bedoya, M., Toro, I. y Arango, B. (2017). Emprendimiento Corporativo e Innovación: Una Revisión y Futuras Líneas de Investigación. Espacios, 38(17), 20-37.

Blesa, A. y Ripolles, M. (2005). Relación entre la orientación al mercado y la orientación emprendedora: su influencia en el rendimiento de la empresa. Revista Europea de Dirección y Economía de la Empresa, 14(3), 165-180.

Blonqvist, K., Hurmelinna, P., Nummela, N. y Saarenketo, S. (2008). The role of trust and contracts in the internationalization of technology intensive Born Globals. Journal of Engineering and Technology Management, 25(12), 123-135.

Calof, J. y Beamish, P. (1995). Adapting to foreing markets: Explaining internalization. International Business Review, 4(2), 115-131.

Cadogan, J. W., Diamantopoulos, A. y De Mortanges, C. P. (1999). A measure of export market orientation: scale development and cross-cultural validation. Journal of International Business Studies, 30(4), 689-707.
Cadogan, J. W., Diamantopoulos, A. y Siguaw, J. A. (2002). Export marketoriented activities: their antecedents and performance consequences. Journal of International Business Studies, 33(3), 615-626.

Covin, J. G. y Slevin O. P. (1991). A conceptual model of entrepreneurship as firm behavior. Entrepreneurship Theory and Practice, 16(1), 7-25.

Desai, P. (2015). Intrapreneurship Promotion. International Journal of Research, 2(2), 1033-1041.

Diamantopolus, A. y Cadogan, J. W. (1996). Internationalizing the market orientation construct: an in-depth interview approach. Journal of Strategic Marketing, 4(1), 23-52.

Flores, A., Ojeda, F., Lee, H. S. y Ramírez, E. (2016). Impacto de la orientación emprendedora en el desempeño empresarial: el caso de las empresas de software en Yucatán, México. FIR -FAEDPYME Intenational Review, 5(9), 25-34.

Ferreira, J. J., Azevedo, G. S. y Fernández, R. (2011). Contribution of Resource-Based View and Entrepreneurial Orientation on Small Firm Growth. Cuadernos de Gestión, 11(1), 95-116.

Gálvez, E. J. y García D. (2011). Impacto de la cultura intraemprendedora en el rendimiento de las MIPYME del sector turístico colombiano: Un estudio empírico. Investigación y Ciencia, (52), 27. 36.

Garzón, M. A. (2004). La innovación intraemprendedora liderada por los gerentes de las Pymes. Universidad y Empresa, 3(6), 74-109.

Giménez, A. O. (2010). La internacionalización de la empresa española y la decisión de exportar como solución a la crisis. Revista de Sociales y Jurídicas, 1(6), 88-111. 
Giménez, A. O. y Piedecausa, J. L. (2015). Plan de internacionalización empresarial. Manual práctico. Madrid, España: Editorial ESIC.

Hair, J., Anderson, R., Tatham, R. y Black, W. (1999). Análisis Multivariante (5 ed.). Madrid: Prentice Hall.

Harveston, P., Ben L. K. y Davis, P. (2000). Internationalization of Born Global and Gradual Globalizing Firms: The Impact of the Manager. Advances in Competitiveness Research, 8(1), 92-99.

Knight, G. y Cavusgil, T. (1996). The born global firm: A challenge to traditional internationalization theory. Advances in International Marketing, 8, 11-26.

Kohli, A. K. y Jaworski, B. J. (1990). Market orientation: the construct, research propositions, and managerial implications. The Journal of Marketing, 54, $1-18$.

Kyriakopoulos, K., Meulenber, M. y Nilsson, J. (2004). The Impact of Cooperative Structure and Firm Culture on Market Orientation and Performance. Agrobusiness, 20(4), 379-396.

Li, Y., Liu Y., Duan, Y. y Li, M. (2008). Entrepreneur Orientation, strategy flexibilities and indigenous firms innovation in transitional china. International journal of technology management, 41(1-2), 223-246.

Lohrke, F. T., Franklin, G. M. y Kothari, V. B. (2015). Top management international orientation and small business exporting performance: The moderating roles of export market y industry factors. Journal of Small Business Strategy, 10(1), 13-24.

Lu, J. W., Liang, X., Shan, M. y Liang, X. (2015). Internationalization and Performance of Chinese Family Firms: The Moderating Role of Corporate Governance. Management and Organization Review, 11(4), 645-678.
Lumpkin, G. T. y Dess, G. G. (1996). Clarifying the Entrepreneurial Orientation Construct and Linking it to Performance. Academy of Management Review, 21(1), 135-172.

Martins, I., Rialp, A., Rialp, J. y AliagaIsla, R. (2015). The Use of Networks to Promote Entrepreneurial Orientation on Small and Medium Company Growth. Innovar, 25(55), 117-130.

McKinsey y Co. (1993). Emerging Exporters. Australia's High Value-Added Manufacturing. Australian Manufacturing Council. Melbourne, Australia.

Madsen, T. K., Rasmussen, E. y Servais, P. (2001). Differences and similarities between born globals and other types of exporters. Advances in international marketing, 10(2), 247-265.

Maldonado, G., García, D., Martínez, M., Aguilera, L., González, M. y Vivanco, S. (2013). Capacidad de internacionalización, actividad innovadora e intraemprendimiento en la MIPYME: Un análisis cross-cultual AguascalientesMurcia. Aguascalientes, México: Editorial Universidad de Aguascalientes.

Matzuno, K. y Mentzer, J. T. (2002). The effects of entrepreneurial proclivity and market orientation on business performance. Journal of marketing, 66(3), 18-32.

Múnera, E. A., Arroyave, S. T., Vanegas, J. G., Morales, J. A. R. y Botero, S. B. (2015). Internacionalización empresarial: características gerenciales y métodos aplicados, una revisión analítica. Global Conference on Business $y$ Finance Proceedings, 10(1), 568576.

Oviatt, B. M. y McDougall, P. P. (1994). Toward a theory of international new ventures. Journal of International Business Studies, 25(1), 45-64. 
Rasmussen, E. y Madsen, T. (2002). The Born Global Concept. Presentación para EIBA 2002 Conference in Athens, Athens, Greece.

Reyes, M. F., Rodríguez, A. y Gálvez, E. J. (2015). Efecto de la actitud de los directivos frente al riesgo en la orientación exportadora de las empresas. Un estudio empírico en Colombia. Cuadernos de Administración, 28(50), 91-112.

Robinson, P. B., Stimpson, D. V., Huefner, J. C. y Hunt, H. K. (1991). An attitude approach to the prediction of entrepreneurship. Entrepreneurship theory and practice, 15(4), 13-31.

Ruesga, S. M. y Bichara, J. D. S. (2007). Competitividad y globalización: nuevos y viejos desafíos. Papeles del Este, 14, 7-27.

Ruzzier, M., Hisrich, R. y Antoncic, B. (2006). SME internationalization research, past, present and future. Journal of Small Business Enterprise Developement, 13(4), 476, 497.

Santos, V. y García, T. (2016). Motivación del empresario y atención informativa en la internacionalización: un análisis regional en el sector español de piedra natural.EuropeanResearchon Management and Business Economics, 22(1), 1-7.

Sharma, D. D. y Blomstermo, A. (2003). The internationalization process of Born Globals: a network review. International Business Review, 12(6), 739-753.

Steenkamp, E. M. y Burgess, S. M. (2006). Marketing renaissance: How research in emerging markets advances marketing science and practice. International Journal of Research in Marketing, 23(4), 337-356.

Trujillo, M. A. y Guzmán, A. (2008). Intraemprendimiento: Una revisión al constructo teórico, sus implicaciones y agenda de investigación futura. Cuadernos de Administración, 21(35), 37-63.
Vanegas, J. G., Restrepo, J. A. y González, M. A. (2015). Negocios y comercio internacional: evidencias de investigación académica para Colombia. Suma de Negocios, 6(13), 84-91.

Varela, J. e Irizar, I. (2009). Caracterización de los Intraemprendimientos en el Grupo Mondragón de España y en las empresas de Ibagué en Colombia. Recuperado el 21 de noviembre de 2017 de: https://www.researchgate.net/profile/ D i a n a _ L o n d o no $6 / \mathrm{publica-}$ tion/38320128_Caracterizacin_de_ los_intraemprendimientos_en_el_ Grupo_Mondragn_de_Espaa_y_en_ las_empresas_de_Ibagu_en_Colombia/links/556c5eff08aeccd7773af5f0. pdf

Wiklund, J. y Shepherd, D. (2005). Entrepreneurial Orientation and Small Business Perfomance: a configurational approach. Journal of Business Venturing, 20, 71-91.

Wood, C. (2004). Entrepreneurial Mindset in Department of Defense (DoD) Organizations Antecedents and Outcomes. [Tesis de maestría]. School of Engineering and Management, Air Force Institute of Technology, Wright-Patterson Air Force Base. Ohio, EEUU. Recuperado el 12 de diciembre del 2016 de: http://www.dtic.mil/dtic/tr/fulltext/u2/ a423134.pdf

Zahra, S., Jennings, D. y Kuratko, D. (1999). The antecedents and consequences of Firm-level Entrepreneurship: The estate of field. Entrepreneurship Theory and Practice, 24(2), 45-65. 
Edgar Julián Gálvez-Albarracín. Administrador de empresas. Magíster en Creación de Empresas y Gestión de Proyectos Innovadores. Doctor en Administración y Dirección de Empresas. Profesor titular de la Facultad de Ciencias de la Administración de la Universidad del Valle, Cali, Colombia.

Miguel Fernando Reyes-Velasco. Administrador de empresas. Especialista en Marketing Estratégico. Magíster en Ciencias de la Organización. Profesor de la Facultad de Ciencias de la Administración de la Universidad del Valle, Cali, Colombia.

Augusto Rodríguez-Orejuela. Ingeniero metalúrgico. Doctor en Ciencias de la Empresa. Profesor titular de la Facultad de Ciencias de la Administración de la Universidad del Valle, Cali, Colombia. 